This item is the archived peer-reviewed author-version of:

The chemical diversity of Lantana camara : analyses of essential oil samples from Cuba, Nepal, and Yemen

\title{
Reference:
}

Satyal Prabodh, Crouch Rebecca A., Monzote Lianet, Cos Paul, Ali Nasser A. Awadh, Alhaj Mehdi A., Setzer William N..- The chemical diversity of Lantana camara : analyses of essential oil samples from Cuba, Nepal, and Yemen

Chemistry \& biodiversity - ISSN 1612-1872 - 13:3(2016), p. 336-342

Full text (Publishers DOI): http://dx.doi.org/doi:10.1002/CBDV.201500271

To cite this reference: http://hdl.handle.net/10067/1315050151162165141 


\section{The Chemical Diversity of Lantana camara: Analyses of Essential Oil Samples from Cuba, Nepal, and Yemen}

by Prabodh Satyal ${ }^{a}$ ), Rebecca A. Crouch ${ }^{a}$ ), Lianet Monzote $^{b}$ ), Paul Cos $^{c}$ ), Nasser A. Awadh Ali $^{\mathrm{d}, \mathrm{e}}$ ), Mehdi A. Alhaj ${ }^{\mathrm{f}}$, and William N. Setzer*a)

a) Department of Chemistry, University of Alabama in Huntsville, Huntsville, AL 35899, USA (phone: +1-256-824-6519; fax: +1-256-824-6349; email: wsetzer@chemistry.uah.edu)

b) Parasitology Department, Institute of Tropical Medicine "Pedro Kouri", 10400 Havana, Cuba (email: monzote@ipk.sld.cu)

c) Laboratory for Microbiology, Parasitology and Hygiene, Faculty of Pharmaceutical, Biomedical and Veterinary Sciences, Antwerp University, Belgium

d) Pharmacognosy Department, Faculty of Clinical Pharmacy, Albaha University, Al Baha, Kingdom of Saudi Arabia

e) Pharmacognosy Department, Faculty of Pharmacy, Sana'a University, P. O. Box 13150, Sana’a, Yemen (email: alinasser9678@yahoo.com)

f) Pharmacognosy Department, Faculty of Pharmacy, Aden University, Aden, Yemen 
Abstract: The aerial parts of Lantana camara L. were collected from three different geographical locations: Artemisa (Cuba), Biratnagar (Nepal), and Sana'a (Yemen). The essential oils were obtained by hydrodistillation and analyzed by gas chromatography - mass spectrometry. A cluster analysis of 39 L. camara essential oil compositions revealed eight major chemotypes: $\beta$-caryophyllene, germacrene $\mathrm{D}$, ar-curcumene/zingiberene, $\gamma$-curcumen-15-al/epi$\beta$-bisabolol, (E)-nerolidol, davanone, eugenol/alloaromadendrene, and carvone. The sample from Cuba falls into the group dominated by $(E)$-nerolidol, the sample from Nepal is a davanone chemotype, and the sample from Yemen belongs to the (E)-caryophyllene chemotype. The chemical composition of L. camara oil plays a role in the biological activity; the $\beta$-caryophyllene and $(E)$-nerolidol chemotypes showed antimicrobial and cytotoxic activities.

Key-words: Essential oil composition, cluster analysis, chemotaxonomy, antimicrobial, cytotoxicity. 
Introduction. - Lantana camara L. (Verbenaceae), is native to the Neotropics but has been introduced to tropical and subtropical locations worldwide where it has become an invasive pest [1,2]. L. camara has been found to be hepatotoxic to grazing animals, attributed to pentacyclic lantadene triterpenoids [3], and human poisoning has also been reported [4]. Nevertheless, L. camara is used as a traditional herbal medicine in many cultures. For example, in Guatemala, Mexico, and Puerto Rico, decoctions of L. camara are taken as a remedy for rheumatism [5]; leaves of L. camara are applied to swollen joints in Sri Lanka [6]; the Malayali people of Chitteri Hills, India, use L. camara leaf juice to treat diabetes [7]; and in Bangladesh, L. camara is used to treat fever and boils [8]. The phytochemistry, toxicology, and medicinal properties of $L$. camara have been extensively reviewed [9-12].

A survey of the literature indicates that there is a great deal of diversity in the composition of the essential oils of $L$. camara growing in different localities, and several chemotypes have been described, including a sabinene/cineole/ $\beta$-caryophyllene chemotype from Nigeria and Iran [13-16]; $\beta$-caryophyllene/ $\gamma$-muurolene/bicyclogermacrene from Costa Rica [17]; germacrene D (15.9\%), $\beta$-caryophyllene (12.4\%), $\alpha$-humulene (9.3\%) from China [18]; $\beta$ caryophyllene, (34.6\%), caryophyllene oxide (10.8\%) from Algeria [19]; $\beta$-caryophyllene (9.8\%), 1,8-cineole (9.4\%) and $\beta$-pinene (8.2\%) from Egypt [20]; davanone (23.5\%), $\beta$ caryophyllene (11.7\%)/sabinene; or davanone $(15 \%) / \beta$-caryophyllene $(12 \%)$ from Madagascar [21,22]; $\beta$-caryophyllene (23.3\%), $\alpha$-humulene (11.5\%), germacrene D (10.9\%) or davanone/ $\beta$ caryophyllene /bicyclogermacrene from India [23,24]; (E)-nerolidol (43.4\%), $\delta$-cadinene (7.6\%), from Cuba [25]; germacrene D (31.0\%) and $\beta$-caryophyllene (14.8\%) from Venezuela [26]; bicyclogermacrene (19.4\%), isocaryophyllene (16.7\%), valencene $(12.9 \%)$ and germacrene D (12.3\%) from Brazil [27]. In this report, we have analyzed the essential oil compositions of $L$. 
camara collected from different geographical locations: Artemisa in Cuba, Biratnagar in Nepal, and Sana'a in Yemen. In addition, we have examined various biological activities of the oils and we have carried out a cluster analysis based on the compositions of 39 different L. camara essential oils.

Results and Discussion. - Essential Oil Compositions. The essential oils from the aerial parts of L. camara from three very different geographical locations, Cuba, Nepal, and Yemen were obtained by hydrodistillation and analyzed by gas chromatography - mass spectrometry (GC-MS). The essential oil compositions are summarized in Table 1.

Lantana camara Oil from Cuba. A total of 112 compounds were identified in the essential oil of L. camara from Cuba, representing $91.2 \%$ of the total oil composition. The major components of the essential oil were $(E)$-nerolidol $(16.6 \%),(E)-\beta$-farnesene $(11.3 \%), \beta$ caryophyllene (9.1\%), germacrene D (7.3\%), and 1,8-cineole (6.9\%). There was also an unidentified component $(\mathrm{RI}=1614,7.8 \%)$. The composition of this L. camara sample from Cuba is qualitatively similar to a previous report of L. camara essential oil from Cuba [25].

Lantana camara Oil from Nepal. A total of 77 compounds were identified in L. camara oil from Nepal, $92.4 \%$ of the composition. The oil from Nepal was dominated by oxygenated sesquiterpenoids (64.0\%), largely davanone (44.4\%), and (E)-nerolidol (13.0\%), with diminutive concentrations of monoterpenoids (total of 5.4\%).

Lantana camara Oil from Yemen. Analysis of lantana oil from Sana'a, Yemen, revealed a total of 71 compounds identified ( $89.4 \%$ of the composition). The oil was dominated by sabinene (16.9\%) and $\beta$-caryophyllene (13.8\%), with lesser concentrations of 1,8-cineole (9.0\%), 
$\alpha$-humulene (5.9\%), and bicyclogermacrene (5.8\%). The Yemeni lantana oil was comparable in composition to samples from Iran [16] and from Nigeria [13,14].

Hierarchical Cluster Analysis. There have been several previous investigations into the chemical diversity of $L$. camara essential oils [17,21,24,28,29], and several chemotypes have been identified (see above). This present study complements previous efforts by including three samples from different geographical locations.

We have carried out a cluster analysis of 35 published [14-18,21-23,25-44] L. camara essential oil compositions in addition to the three samples in this present study (see Fig. 1). The cluster analysis reveals eight different chemotypes: (A) a $\beta$-caryophyllene chemotype, (B) a germacrene D chemotype, (C) an ar-curcumene/ $\alpha$-zingiberene chemotype, (D) a $\gamma$-curcumen-15al/epi- $\beta$-bisabolene chemotype, $(\mathbf{E})$ an $(E)$-nerolidol chemotype, $(\mathbf{F})$ a davanone chemotype, $(\mathbf{G})$ a eugenol/alloaromadendrene chemotype, and $(\mathbf{H})$ a carvone chemotype. The cluster analysis places the essential oil from Yemen in the $\beta$-caryophyllene-rich cluster, which is also the most prominent chemotype. The L. camara oil from Nepal represents the davanone-rich chemotype, while the sample from Cuba, along with the previous Cuban lantana oil, are in the (E)-nerolidol cluster. The chemical diversity displayed by L. camara essential oils cannot be attributed to geographical location. Thus, for example, samples from Brazil are represented in four different chemotypes and lantana oils from India are found in three chemotypes. The chemical diversity of L. camara may be due to the numerous different cultivars and hybrids [2,9]. The Missouri Botanical Garden currently lists 47 different varieties and subspecies of L. camara [45].

Bioactivity Screening. Antimicrobial assessment of essential oil from L. camara collected in Cuba was performed fluorimetrically after adding resazurin. The essential oil was inactive against Escherichia coli and Candida albicans, with $\mathrm{IC}_{50}$ values $>64 \mu \mathrm{g} / \mathrm{mL}$; while an 
$\mathrm{IC}_{50}$ of $12.13 \mu \mathrm{g} / \mathrm{mL}$ was obtained against Staphylococcus aureus. However, cytotoxicity displayed against MRC-5 cells was higher, with an $\mathrm{IC}_{50}$ value of $10.31 \mu \mathrm{g} / \mathrm{mL}$. The $L$. camara oil from Nepal was screened for antibacterial activity against Bacillus cereus, S. aureus, E. coli, and Pseudomonas aeruginosa but was inactive (MIC $>1250 \mu \mathrm{g} / \mathrm{mL}$ ) against all bacteria tested. The Nepalese L. camara oil was also inactive against C. albicans (MIC $=1250 \mu \mathrm{g} / \mathrm{mL}$ ) and Aspergillus niger $(\mathrm{MIC}=625 \mu \mathrm{g} / \mathrm{mL}$ ). L. camara oil from Nepal was tested for brine shrimp (Artemia salina) lethality and nematicidal (Caenorhabditis elegans) activity, but was only marginally active $\left(\mathrm{LC}_{50}=24.9\right.$ and $117.5 \mu \mathrm{g} / \mathrm{mL}$, respectively). In-vitro cytotoxicity screening against MCF-7 human ductal carcinoma cells also showed marginal activity (only $24.1 \%$ kill at $100 \mu \mathrm{g} / \mathrm{mL})$.

Lantana oil from Yemen was screened for antimicrobial activity using the disc diffusion assay against $S$. aureus, methicillin-resistant S. aureus (MRSA), Bacillus subtilis, E. coli, P. aeruginosa, and C. albicans. In this assay, S. aureus, B. subtilis, and C. albicans were more susceptible to the oil, with inhibition zones ranging from $26 \pm 2.8$ to $38 \pm 3.6 \mathrm{~mm}$. The essential oil of Yemeni L. camara showed similarities in its chemical composition with the Nigerian essential oil [13] and both oils exhibited antimicrobial activity. L. camara essential oils from India $[38,44]$ also showed antimicrobial activity, and these oils also belong to the $\beta$ caryophyllene-rich chemotype. Notably, the lantana oil from Nepal (davanone chemotype) did not exhibit biological activity and the oil from Venezuela (germacrene D chemotype) [26] showed only marginal antibacterial activity. Similarly, lantana oils from Brazil ( $\beta$-caryophyllene chemotype) [42] and from Cuba [(E)-nerolidol chemotype] showed cytotoxic activity, while the oil from Nepal was inactive. Consistent with these results, $\beta$-caryophyllene has shown both 
antimicrobial [46] and cytotoxic [47-49] activities. Likewise, (E)-nerolidol has exhibited antibacterial $[50,51]$ and cytotoxic $[47,52]$ effects.

Conclusions. - Lantana camara essential oils show a wide diversity of chemical composition with at least eight chemotypes. The particular chemotype of L. camara may be important with respect to the use of this plant in herbal medicine. The $\beta$-caryophyllene and the (E)-nerolidol chemotypes have displayed biological activities while the davanone or the germacrene D chemotypes are less active. The chemical compositions of essential oils, particularly those with wide chemical variability such as $L$. camara, should be considered when assessing the bioactivities.

P.S. thanks Dr. Ghanashyam Shrivastav for providing access to laboratory facilities in Nepal and Dr. Bernhard Vogler for technical assistance with GC-MS data collection. W.N.S. is grateful to an anonymous private donor for the generous gift of the GC-MS instrumentation.

\section{Experimental Part}

Plant Material and Essential Oils. Aerial parts of L. camara were collected from Artemisa in Cuba $\left(22^{\circ} 49^{\prime} \mathrm{N}, 82^{\circ} 45^{\prime} \mathrm{W}, 43 \mathrm{~m}\right.$ above sea level), in October 2014 . The plant was identified by Eldys Bécquer Granados from the National Botanical Garden, Havana, Cuba, where a voucher specimen was deposited. The plant sample was crushed and the essential oil was obtained by hydrodistilled using a Clevenger type apparatus for $5 \mathrm{~h}$ to give a light-yellow-colored essential oil with a yield of $0.3 \%$. 
Aerial parts of L. camara were collected from city of Biratnagar $\left(26^{\circ} 28^{\prime} \mathrm{N}, 87^{\circ} 16^{\prime} \mathrm{E}\right.$, and $1072 \mathrm{~m}$ above sea level) in Morang district in Koshi Zone in Nepal in May 2011. The plant material was identified by Tilak Gautam, and a voucher specimen has been deposited in the herbarium of the Tribhuvan University, Post-Graduate Campus, Botany Department, Biratnagar. The essential oil was extracted from fresh aerial samples (100 g) that were crushed and hydrodistilled using a Clevenger type apparatus for $4 \mathrm{~h}$. The clear pale yellow essential oil $(0.7$ g) produced was stored at $4^{\circ} \mathrm{C}$ until analyzed.

The aerial parts of L. camara were collected in the early morning from Botanical garden, Faculty of Pharmacy, Sana'a University, Yemen, in November, 2010. The plant was identified by Hassan M. Ibrahim of the Botany Department, Faculty of Sciences, Sana'a University. A voucher specimen of the plant material (YMP-Verben.-1) has been deposited at the Pharmacognosy Department, Sana'a University, Yemen. The plant sample (100 g) was hydrodistilled using a Clevenger apparatus for $3 \mathrm{~h}$ to give colorless oil, $0.5 \% \mathrm{w} / \mathrm{w}$ yield. After separation of the obtained essential oil, it was dried over anhydrous sodium sulfate.

Gas Chromatographic - Mass Spectral Analysis. Gas chromatographic - mass spectral analyses was performed on the essential oils of $O$. basilicum using an Agilent $6890 \mathrm{GC}$ with Agilent 5973 mass selective detector and a fused silica capillary column (HP $5 \mathrm{~ms}, 30 \mathrm{~m} \times 0.25$ $\mathrm{mm})$ coated with $5 \%$ phenyl-polymethylsiloxane $(0.25 \mu \mathrm{m}$ phase thickness $)$ as previously described [53]. Identification of the oil components was based on their Kovats retention indices determined by reference to a homologous series of $n$-alkanes, and by comparison of their mass spectral fragmentation patterns with those reported in the literature [54], and stored on the MS library [NIST database (G1036A revision D.01.00)/ChemStation data system (G1701CA, 
version C.00.01.080)]. The percentages of each component are reported as raw percentages based on total ion current without standardization. The chemical compositions of the L. camara oils are summarized in Table 1.

Cytotoxicity Assays. Cell culture experiments were performed with MCF-7 (human mammary ductal carcinoma) using the MTT assay, as described previously [53].

Antimicrobial Assays. Antibacterial screening against Bacillus cereus, Bacillus subtilis, Escherichia coli, Pseudomonas aeruginosa, Staphylococcus aureus, and methicillin-resistant $S$. aureus (MRSA), and antifungal screening against Aspergillus niger were carried out using the resazurin fluorometric assay [55], the microbroth dilution assay [56] or the disc diffusion assay [57] as previously described.

Invertebrate Toxicity Assays. Nematicidal (Caenorhabditis elegans) activity and brine shrimp (Artemia salina) lethality evaluations were carried out as previously described [58].

Hierarchical Cluster Analysis. A total of 36 L. camara essential oil compositions from the published literature, as well as the compositions from this study were treated as operational taxonomic units (OTUs). The percentage composition of 37 major essential oil components [ $\beta$ caryophyllene, germacrene D, bicyclogermacrene, sabinene, $\alpha$-humulene, davanone, 1,8-cineole, (E)-nerolidol, ar-curcumene, carvone, valencene, spathulenol, caryophyllene oxide, limonene, $\beta$ elemene, germacrene B, $\alpha$-pinene, $\delta$-3-carene, $\beta$-pinene, linalool, $\alpha$-phellandrene, alloaromadendrene, $\beta$-bisabolene, $\alpha$-zingiberene, $\delta$-cadinene, $\alpha$-copaene, $\gamma$-terpinene, $\gamma$-cadinene, $\gamma$-elemene, $\gamma$-curcumene, myrcene, eugenol, $\tau$-cadinol, $\gamma$-curcumen-15-al, epi- $\beta$-bisabolol, $\alpha$ cadinol, and cubebol] was used to determine the chemical relationship between the various $L$. camara essential oil samples by agglomerative hierarchical cluster (AHC) analysis using the XLSTAT software, version 2015.4.01. Pearson correlation was selected as a measure of 
similarity, and the unweighted pair-group method with arithmetic average (UPGMA) was used for cluster definition. The resulting dendrogram is shown in Figure 1. 


\section{REFERENCES}

[1] G. P. Sharma, A. S. Raghubanshi, J. S. Singh, Weed Biol. Manag. 2005, 5, 157.

[2] S. A. Bhagwat, E. Breman, T. Thekaekara, T. F. Thornton, K. J. Willis, PLoS ONE 2012, 7(3), e32407.

[3] O. P Sharma, S. Sharma, V. Pattabhi, S. B. Mahato, P. D. Sharma, Crit. Rev. Toxicol. $\mathbf{2 0 0 7}, 37,313$.

[4] S. L. Wolfson, T. W. G. Solomons, JAMA Pediatrics 1964, 107, 173.

[5] J. F. Morton, Atlas of Medicinal Plants of Middle America, Vol II. Charles C. Thomas, Publisher, Springfield, Illinois, USA, 1981, p. 739.

[6] E. R. H. S. S. Ediriweera, Trop. Agric. Res. Exten. 2007, 10, 11.

[7] K. Kadhirvel, S. Ramya, T. Palin Sathya Sudha, A. Veera Ravi, C. Rajasekaran, R. Vanitha Selvi, R. Jayakumararaj, Environ. We Int. J. Sci. Technol. 2010, 5, 35.

[8] M. Rahmatullah, R. Jahan, S. Azam, S. Hossan, M. A. H. Mollik, T. Rahman, Afr. J. Trad. Complement. Altern. Med. 2011, 8(S), 53.

[9] E. L. Ghisalberti, Fitoterapia 2000, 71, 467.

[10] S. Patel S, Rev. Environ. Sci. Tiotechnol. 2011, 10, 341.

[11] S. Kalita, G. Kumar, L. Karthik, K. V. B. Rao, Res. J. Pharm. Tech. 2012, 5, 711.

[12] M. Saxena, J. Saxena, S. Khare, Int. J. Pharm. Life Sci. 2012, 3, 1551.

[13] A. A. Kasali, O. Ekundayo, C. Paul, W. A. Koenig, A. O. Eshilokun, P. Yadua, J. Essent. Oil Res. 2004, 16, 582.

[14] O. O. Sonibare, I. Effiong, Afr. J. Biotechnol. 2008, 7, 2618.

[15] O. A. Oyedeji, O. Ekundayo, W. A. König, W. A. Flavour Fragr. J. 2003, 18, 384.

[16] F. Sefidkon, Flavour Fragr. J. 2002, 17, 78. 
[17] A. B. Walden, W. A. Haber, W. N. Setzer, Nat. Prod. Commun. 2009, 4, 105.

[18] A. J. Sundufu, H. Shoushan, Flavour Fragr. J. 2004, 19, 229.

[19] S. Zoubiri, A. Baaliouamer, Vet. Parasitol. 2011, 181, 370.

[20] N. M. Abdel-Hady, A. S. Abdei-Halim, A. M. Al-Ghadban, J. Egypt Soc. Parasitol. $\mathbf{2 0 0 5}, 35,687$.

[21] J.-A. Randrianalijaona, P. A. R. Ramanoelina, J. R. E. Rasoarahona, E. M. Gaydou, J. Essent. Oil Res. 2006, 18, 405.

[22] M. B. Ngassoum, S. Yonkeu, L. Jirovetz, G. Buchbauer, G. Schmaus, F.-J. Hammerschmidt, Flavour Fragr. J. 1999, 14, 245.

[23] V. S. Rana, D. Prasad, M. Amaro Blazquez, J. Essent. Oil Res. 2005, 17, 198.

[24] L. Misra, A. K. Saikia, J. Essent. Oil Res. 2011, 23, 1.

[25] J. A. Pino, R. Marbot, A. Rosado, C. Romeu, M. P. Mart, J. Essent. Oil Res. 2004, 16, 216.

[26] N. R. Tesch, F. Mora, L. Rojas, T. Díaz, J. Velasco, C. Yánez, N. Rios, J. Carmona, S. Pasquale, Nat. Prod. Commun. 2011, 6, 1031.

[27] E. O. Sousa, N. F. Silva, F. F. Rodrigues, A. R. Campos, S. G. Lima, J. G. Costa, Pharmacogn. Mag. 2010, 6(22), 79.

[28] M. J. L. da Silva, E. H. A. Andrade, M. d. G. B. Zoghbi, A. I. R. Luz, J. D. da Silva, J. G. S. Maia, Flavour Fragr. J. 1999, 14, 208.

[29] J. Benites, C. Moiteiro, G. Miguel, L. Rojo, J. López, F. Venâncio, L. Ramalho, S. Feio, S. Dandlen, H. Casanova, I. Torres, J. Chil. Chem. Soc. 2009, 54, 379.

[30] S. Möllenbeck, T. König, P. Schreier, W. Schwab, J. Rajaonarivony, L. Ranarivelo, Flavour Fragr. J. 1997, 12, 63. 
[31] P. Weyerstahl, H. Marschall, A. Eckhardt, C. Christiansen, Flavour Fragr. J. 1999, 14, 15.

[32] M. Khan, S. K. Strivastava, K. V. Syamasundar, M. Singh, A. A. Naqvi, Flavour Fragr. J. 2002, 17, 75 .

[33] M. I. E. Mohamed, S. A. M. Abdelgaleil, Appl. Entomol. Zool. 2008, 43, 599.

[34] M. Vergeguer, M. A. Blázquez, H. Boira, Biochem. Systemat. Ecol. 2009, 37, 362.

[35] E. O. Sousa, A. V. Colares, F. F. G. Rodrigues, A. R. Campos, S. G. Lima, J. G. M. Costa, Rec. Nat. Prod. 2010, 4, 31.

[36] R. C. Padalia, R. S. Verma, V. Sundaresan, Rec. Nat. Prod. 2010, 4, 109.

[37] J. G. M. Costa, E. F. G. Rodrigues, E. O. Sousa, D. M. S. Junior, A. R. Campos, H. D. M. Coutinho, S. G. de Lima, Chem. Nat. Compd. 2010, 46, 313.

[38] A. K. Saikia, R. K. Sahoo, Middle-East J. Sci. Res. 2011, 8, 599.

[39] H. O. Elansary, M. Z. M. Salem, N. A. Ashmawy, M. M. Yacout, J. Agric. Sci. 2012, 4, 144.

[40] E. O. Sousa, T. S. Almeida, I. R. A. Menezes, F. F. G. Rodrigues, A. R. Campos, S. G. Lima, J. G. M. da Costa, Rec. Nat. Prod. 2012, 6, 144.

[41] S. Zoubriri, A. Baaliouamer, J. Saudi Chem. Soc. 2012, 16, 291.

[42] L. B. P. Medeiros, M. d. S. Rocha, S. G. de Lima, G. R. d. S. Júnior, A. M. d. G. L. Citó, D. da Silva, J. A. D. Lopes, D. J. Moura, J. Saffi, M. Mobin, J. G. M. da Costa, Rev. Bras. Farmacog. 2012, 22, 1259.

[43] S. Murugesan, C. Rajeshkannan, D. Suresh Babu, R. Sumathi, P. Manivachakam, Adv. Appl. Sci. Res. 2012, 3, 2754. 
[44] R. Seth, M. Mohan, P. Singh, S. Z. Haider, S. Gupta, I. Bajpai, D. Singh, R. Dobhal, Asian Pacific J.Trop. Biomed. 2012, S1407.

[45] Tropicos.org. Missouri Botanical Garden. 16 Aug 2015 http://www.tropicos.org/Name/33700010.

[46] J. M. Schmidt, J. A. Noletto, B. Vogler, W. N. Setzer, J. Herbs Spices Med. Plants 2007, 12(3), 43.

[47] I. Kubo, Y. Morimitsu, J. Agric. Food Chem. 1995, 43, 1626.

[48] S. L. da Silva, P. M. Figueiredo, T. Yano, Acta Amazonica 2007, 37, 281.

[49] M. C. Palazzo, H. L. Wright, B. R. Agius, B. S. Wright, D. M. Moriarity, W. A. Haber, W. N. Setzer, Rec. Nat. Prod. 2009, 3, 153.

[50] I. Kubo, H. Muroi, A. Kubo, J. Agric. Food. Chem. 1993, 41, 2447.

[51] Y. Inoue, A. Shiraishi, T. Hada, K. Hirose, H. Hamashima, J. Shimada, FEMS Microbiol. Lett. 2004, 237, 325.

[52] M. Sylvestre, A. Pickette, S. Lavoie, A. Longtin, J. Legault, Phytother. Res. 2007, 21, 536.

[53] F. S. Sharopov, M. Wink, D. R. Khalifaev, H. Zhang, N. S. Dosoky, W. N. Setzer, Am. J. Essent. Oils Nat. Prod. 2013, 1(1), 11.

[54] R. P. Adams, 'Identification of Essential Oil Components by Gas Chromatography / Mass Spectrometry’, 4th Ed., Allured, Carol Stream, Illinois, 2007.

[55] L. Monzote, O. Cuesta-Rubio, A. Matheeussen, T. Van Assche, L. Maes, Cos, Phytother. Res. 2011, 25,458.

[56] M. S. Owolabi, O. A. Lawal, N. S. Dosoky, P. Satyal, W. N. Setzer, Am. J. Essent. Oils Nat. Prod. 2013, 1(1), 4. 
[57] N. A. A. Ali, F. S. Sharopov, M. Alhaj, G. M. Hill, A. Porzel, N. Arnold, W. N. Setzer, J. Schmidt, L. Wessjohann, Nat. Prod. Commun. 2012, 7, 257.

[58] P. Satyal, P. Paudel, A. Poudel, N. S. Dosoky, K. K. Pokharel, W. N. Setzer, Nat. Prod. Commun. 2013, 8, 1777. 
Table 1. Essential oil compositions of Lantana camara from Cuba, Nepal, and Yemen.

\begin{tabular}{|c|c|c|c|c|}
\hline \multirow{2}{*}{$\mathrm{RI}^{\mathrm{a}}$} & \multirow{2}{*}{ Compound } & \multicolumn{3}{|c|}{$\%$ Composition } \\
\hline & & Cuba & Nepal & Yemen \\
\hline 831 & Furfural & - & 0.1 & - \\
\hline 854 & $(2 E)$-Hexenal & - & - & $\operatorname{tr}^{\mathrm{b}}$ \\
\hline 880 & Isoamyl acetate & - & - & $\operatorname{tr}$ \\
\hline 882 & 2-Butylfuran & $\operatorname{tr}$ & - & - \\
\hline 924 & $\alpha$-Thujene & 0.1 & - & 0.4 \\
\hline 931 & $\alpha$-Pinene & 3.2 & - & 2.8 \\
\hline 952 & Camphene & 1.5 & - & 1.0 \\
\hline 961 & Benzaldehyde & - & $\operatorname{tr}$ & - \\
\hline 973 & 3-methylnonane & - & 0.1 & - \\
\hline 976 & Sabinene & 2.0 & $\operatorname{tr}$ & 16.9 \\
\hline 979 & $\beta$-Pinene & 2.5 & - & 1.9 \\
\hline 981 & 1-Octen-3-ol & 0.5 & 5.3 & 0.5 \\
\hline 984 & 3-Octanone & $\operatorname{tr}$ & - & - \\
\hline 992 & 1-Decene & - & $\operatorname{tr}$ & - \\
\hline 992 & Myrcene & 0.5 & - & 1.2 \\
\hline 995 & 3-Octanol & 0.2 & 0.2 & $\operatorname{tr}$ \\
\hline 1000 & $n$-Decane & - & 1.1 & - \\
\hline 1003 & $\alpha$-Phellandrene & 0.1 & - & 0.3 \\
\hline 1008 & (3Z)-Hexenyl acetate & - & $\operatorname{tr}$ & - \\
\hline 1010 & $\delta$-3-Carene & - & - & 2.4 \\
\hline 1016 & $\alpha$-Terpinene & - & - & 0.1 \\
\hline 1019 & 3-Methyl-1,2-cyclopentanedione & $\operatorname{tr}$ & - & - \\
\hline 1024 & $p$-Cymene & 1.3 & - & 0.4 \\
\hline 1028 & Limonene & 1.3 & 0.1 & 1.0 \\
\hline 1029 & $\beta$-Phellandrene & 0.3 & - & - \\
\hline 1031 & 1,8-Cineole & 6.9 & 0.3 & 9.0 \\
\hline
\end{tabular}




\begin{tabular}{|c|c|c|c|c|}
\hline \multirow{2}{*}{$\mathrm{RI}^{\mathrm{a}}$} & \multirow{2}{*}{ Compound } & \multicolumn{3}{|c|}{$\%$ Composition } \\
\hline & & Cuba & Nepal & Yemen \\
\hline 1033 & Benzyl Alcohol & - & 0.4 & - \\
\hline 1038 & $(Z)-\beta$-Ocimene & $\operatorname{tr}$ & - & 0.8 \\
\hline 1049 & $(E)-\beta$-Ocimene & 0.2 & - & 1.1 \\
\hline 1051 & cis-Arbusculone & - & 0.1 & - \\
\hline 1058 & $\gamma$-Terpinene & 0.1 & - & 0.5 \\
\hline 1064 & trans-Arbusculone & - & $\operatorname{tr}$ & - \\
\hline 1068 & cis-Sabinene hydrate & $\operatorname{tr}$ & 0.2 & 1.2 \\
\hline 1072 & cis-Linalool oxide (furanoid) & - & 0.1 & - \\
\hline 1087 & Terpinolene & $\operatorname{tr}$ & - & 0.3 \\
\hline 1097 & trans-Sabinene hydrate & - & 0.1 & 0.5 \\
\hline 1099 & Linalool & 0.9 & 1.2 & 0.3 \\
\hline 1103 & 2-Methylbutyl 2-methylbutyrate & 0.1 & - & 0.4 \\
\hline 1109 & 2-Methylbutyl isovalerate & - & - & $\operatorname{tr}$ \\
\hline 1112 & endo-Fenchol & - & - & 0.2 \\
\hline 1113 & $\alpha$-Fenchocamphorone & $\operatorname{tr}$ & - & - \\
\hline 1115 & trans-Thujone & - & - & $\operatorname{tr}$ \\
\hline 1120 & cis-p-Menth-2-en-1-ol & $\operatorname{tr}$ & - & 0.1 \\
\hline 1125 & $\alpha$-Campholenal & - & - & $\operatorname{tr}$ \\
\hline 1139 & trans-Sabinol & $\operatorname{tr}$ & - & - \\
\hline 1140 & cis-Verbenol & - & $\operatorname{tr}$ & - \\
\hline 1141 & trans-p-Menth-2-en-1-ol & $\operatorname{tr}$ & - & - \\
\hline 1144 & trans-Verbenol & 0.1 & - & - \\
\hline 1145 & Camphor & 0.9 & 0.5 & 1.5 \\
\hline 1151 & Lilac aldehyde D & - & 0.1 & - \\
\hline 1153 & Camphene hydrate & $\operatorname{tr}$ & - & - \\
\hline 1157 & $\beta$-Pinene oxide & - & 0.2 & - \\
\hline 1162 & Isoborneol & 0.5 & - & 0.4 \\
\hline
\end{tabular}




\begin{tabular}{|c|c|c|c|c|}
\hline \multirow{2}{*}{$\mathrm{RI}^{\mathrm{a}}$} & \multirow{2}{*}{ Compound } & \multicolumn{3}{|c|}{$\%$ Composition } \\
\hline & & Cuba & Nepal & Yemen \\
\hline 1165 & $\delta$-Terpineol & 0.1 & - & 0.1 \\
\hline 1170 & Borneol & 0.3 & 0.9 & - \\
\hline 1171 & (2E)-Nonenol & - & 0.2 & - \\
\hline 1174 & cis-Pinocamphone & $\operatorname{tr}$ & - & - \\
\hline 1176 & Terpinen-4-ol & 0.5 & 0.3 & 0.8 \\
\hline 1185 & $p$-Cymen-8-ol & $\operatorname{tr}$ & - & - \\
\hline 1189 & $\alpha$-Terpineol & 1.7 & 0.2 & 0.2 \\
\hline 1192 & Methyl salicylate & - & 0.7 & - \\
\hline 1200 & Dodecane & - & 2.3 & - \\
\hline 1205 & Verbenone & $\operatorname{tr}$ & 0.2 & - \\
\hline 1229 & Nordavanone & - & $\operatorname{tr}$ & - \\
\hline 1230 & (3Z)-Hexenyl 2-methylbutanoate & $\operatorname{tr}$ & - & - \\
\hline 1237 & (2Z)-Hexenyl isovalerate & $\operatorname{tr}$ & - & - \\
\hline 1251 & Piperitone & 0.6 & - & - \\
\hline 1282 & Bornyl acetate & $\operatorname{tr}$ & - & - \\
\hline 1288 & Davanone A & - & 0.1 & - \\
\hline 1289 & Thymol & $\operatorname{tr}$ & - & - \\
\hline 1333 & $\alpha$-Terpinyl acetate & 0.1 & - & - \\
\hline 1337 & $\delta$-Elemene & - & - & 0.1 \\
\hline 1345 & $\alpha$-Cubebene & $\operatorname{tr}$ & - & - \\
\hline 1349 & 4'-Methoxyacetophenone & - & 0.2 & - \\
\hline 1355 & Eugenol & - & 1.7 & - \\
\hline 1366 & Cyclosativene & $\operatorname{tr}$ & - & - \\
\hline 1375 & $\alpha$-Copaene & 1.5 & 0.5 & 0.6 \\
\hline 1384 & $\beta$-Bourbonene & 0.3 & - & 0.2 \\
\hline 1385 & $\beta$-Cubebene & 0.3 & 0.1 & 0.3 \\
\hline 1392 & $\beta$-Elemene & 1.4 & - & 0.6 \\
\hline
\end{tabular}




\begin{tabular}{|c|c|c|c|c|}
\hline \multirow{2}{*}{$\mathrm{RI}^{\mathrm{a}}$} & \multirow{2}{*}{ Compound } & \multicolumn{3}{|c|}{$\%$ Composition } \\
\hline & & Cuba & Nepal & Yemen \\
\hline 1400 & Tetradecane $+\alpha$-Funebrene & $\operatorname{tr}$ & - & - \\
\hline 1409 & Davanol isomer A & - & 0.1 & - \\
\hline 1419 & $\beta$-Caryophyllene & 9.1 & 2.9 & 13.8 \\
\hline 1429 & $\beta$-Copaene & 1.2 & 0.2 & 0.6 \\
\hline 1431 & trans- $\alpha$-Bergamotene & $\operatorname{tr}$ & - & - \\
\hline 1432 & $\alpha$-Guaiene & 0.2 & - & - \\
\hline 1434 & $\gamma$-Elemene & - & - & $\operatorname{tr}$ \\
\hline 1438 & 6,9-Guaidiene & 0.1 & - & - \\
\hline 1441 & cis-Muurola-3,5-diene & $\operatorname{tr}$ & - & - \\
\hline 1446 & $(Z)-\beta$-Farnesene & 0.1 & - & - \\
\hline 1455 & $\alpha$-Humulene & - & 3.4 & 5.9 \\
\hline 1457 & $(E)-\beta$-Farnesene & 11.3 & $\operatorname{tr}$ & - \\
\hline 1458 & Alloaromadendrene & 0.6 & - & 0.3 \\
\hline 1460 & cis-Cadina-1(6),4-diene & $\operatorname{tr}$ & - & - \\
\hline 1464 & 9-epi-(E)-Caryophyllene & $\operatorname{tr}$ & - & - \\
\hline 1474 & Dodecanol & - & 0.1 & - \\
\hline 1476 & trans-Cadina-1(6),4-diene & 0.4 & 0.1 & - \\
\hline 1477 & trans-4,10-Epoxyamorphane & $\operatorname{tr}$ & - & - \\
\hline 1477 & $\gamma$-Muurolene & & - & 0.3 \\
\hline 1482 & Germacrene D & 7.3 & 0.6 & 2.1 \\
\hline 1485 & $\beta$-Selinene & 0.6 & - & - \\
\hline 1487 & $(E)-\beta$-Ionone & - & - & $\operatorname{tr}$ \\
\hline 1491 & trans-Muurola-4(14),5-diene & 0.1 & - & $\operatorname{tr}$ \\
\hline 1492 & $\alpha$-Selinene & 1.7 & - & - \\
\hline 1494 & epi-Cubebol & - & 0.2 & $\operatorname{tr}$ \\
\hline 1499 & Bicyclogermacrene & - & 0.1 & 5.8 \\
\hline 1500 & Pentadecane & $\operatorname{tr}$ & $\operatorname{tr}$ & - \\
\hline
\end{tabular}




\begin{tabular}{|c|c|c|c|c|}
\hline \multirow{2}{*}{$\mathrm{RI}^{\mathrm{a}}$} & \multirow{2}{*}{ Compound } & \multicolumn{3}{|c|}{$\%$ Composition } \\
\hline & & Cuba & Nepal & Yemen \\
\hline 1501 & $\alpha$-Muurolene & 0.7 & 0.3 & 0.5 \\
\hline 1508 & $\alpha$-Bulnesene & $\operatorname{tr}$ & - & - \\
\hline 1506 & Isodavanone & - & $\operatorname{tr}$ & - \\
\hline 1506 & Germacrene A & $\operatorname{tr}$ & - & 0.5 \\
\hline 1509 & $\beta$-Bisabolene & 0.7 & 0.1 & - \\
\hline 1509 & $\delta$-Amorphene & 0.1 & - & - \\
\hline 1516 & Cubebol & 1.0 & - & 1.4 \\
\hline 1518 & trans-Calamenene & 0.1 & - & - \\
\hline 1521 & $\beta$-Sesquiphellandrene & 0.1 & - & - \\
\hline 1523 & $\delta$-Cadinene & 0.9 & 0.3 & 0.3 \\
\hline 1533 & Davana ether & - & - & 0.1 \\
\hline 1534 & (Z)-Nerolidol & 0.1 & 0.1 & - \\
\hline 1538 & $\alpha$-Calacorene & 0.1 & - & - \\
\hline 1540 & $\alpha$-Copaene-11-ol & - & 0.2 & - \\
\hline 1545 & Elemol & 0.1 & - & - \\
\hline 1547 & (Z)-Caryophyllene oxide & 0.2 & - & - \\
\hline 1550 & Elemol & - & 0.2 & - \\
\hline 1556 & Germacrene B & 0.5 & 0.2 & 0.8 \\
\hline 1559 & Davanone C & - & 0.3 & - \\
\hline 1559 & Isodavanone & - & - & 0.6 \\
\hline 1560 & $(E)$-Nerolidol & 16.6 & 13.0 & 2.3 \\
\hline 1562 & 7-Hydroxyfarnesene & 0.1 & - & - \\
\hline 1566 & Palustrol & $\operatorname{tr}$ & - & - \\
\hline 1569 & Longipinanol & $\operatorname{tr}$ & - & - \\
\hline 1573 & Davanone B & - & 0.4 & 0.3 \\
\hline 1576 & Germacrene D-4-ol & - & 0.3 & $\operatorname{tr}$ \\
\hline 1580 & Spathulenol & 0.7 & - & 2.2 \\
\hline
\end{tabular}




\begin{tabular}{|c|c|c|c|c|}
\hline \multirow{2}{*}{$\mathrm{RI}^{\mathrm{a}}$} & \multirow{2}{*}{ Compound } & \multicolumn{3}{|c|}{$\%$ Composition } \\
\hline & & Cuba & Nepal & Yemen \\
\hline 1583 & Caryophyllene oxide & 2.3 & 0.7 & 2.2 \\
\hline 1584 & Gleenol & 0.1 & - & - \\
\hline 1588 & Salvial-4(14)-en-1-one & 0.1 & - & - \\
\hline 1588 & Davanone & - & 44.4 & 0.4 \\
\hline 1590 & Viridiflorol & 0.6 & - & - \\
\hline 1595 & Fokienol & 0.2 & - & - \\
\hline 1600 & Ledol & 0.1 & - & - \\
\hline 1605 & Humulene epoxide II & 0.5 & - & - \\
\hline 1607 & 1,10 di-epi-Cubenol & - & 0.1 & - \\
\hline 1609 & Unidentified & - & - & 1.4 \\
\hline 1610 & Humulene epoxide II & - & 0.9 & 0.4 \\
\hline 1614 & Unidentified & 7.8 & - & - \\
\hline 1624 & Isospathulenol & 0.4 & - & - \\
\hline 1626 & Eremoligenol & - & 0.1 & - \\
\hline 1631 & Unidentified & 1.0 & 3.3 & 3.5 \\
\hline 1633 & Caryophylla-4(12),8(13)-dien-5 $\alpha$-ol & 0.2 & - & 0.2 \\
\hline 1636 & Caryophylla-4(12),8(13)-dien-5 $\beta$-ol & - & 0.2 & 0.3 \\
\hline 1638 & $\alpha-$ Muurolol (= Torreyol) & 0.4 & - & - \\
\hline 1639 & Unidentified & - & - & 0.7 \\
\hline 1640 & $\tau$-Cadinol & - & 0.8 & 0.5 \\
\hline 1641 & $\tau$-Muurolol & 0.2 & - & - \\
\hline 1643 & $\delta$-Cadinol & 0.3 & - & - \\
\hline 1647 & $\alpha-$ Muurolol & - & - & $\operatorname{tr}$ \\
\hline 1648 & 15-Copaenol & - & 0.3 & - \\
\hline 1651 & $\alpha$-Cadinol & 0.6 & - & - \\
\hline 1652 & Unidentified & - & - & 1.9 \\
\hline 1654 & Selin-11-en-4 $\alpha$-ol & 0.3 & - & - \\
\hline
\end{tabular}




\begin{tabular}{|c|c|c|c|c|}
\hline \multirow{2}{*}{$\mathrm{RI}^{\mathrm{a}}$} & \multirow{2}{*}{ Compound } & \multicolumn{3}{|c|}{$\%$ Composition } \\
\hline & & Cuba & Nepal & Yemen \\
\hline 1661 & trans-Calamenen-10-ol $+\beta$-Atlantone & $\operatorname{tr}$ & - & - \\
\hline 1670 & 14-Hydroxy-9-epi-(E)-caryophyllene & - & 0.7 & - \\
\hline 1677 & Unidentified & - & - & 2.9 \\
\hline 1679 & Khusinol & - & 0.1 & - \\
\hline 1686 & Germacra-4(15),5,10(14)-trien-1 $\alpha$-ol & 0.3 & 0.2 & 0.2 \\
\hline 1686 & $\alpha$-Bisabolol & 0.1 & - & - \\
\hline 1688 & Shyobunol & - & 0.1 & 0.2 \\
\hline 1689 & Eudesma-4(15),7-dien-1 $\beta$-ol & 0.1 & - & - \\
\hline 1687 & Dodecyl acetate & - & 0.1 & - \\
\hline 1697 & Eudesm-7(11)-en-4-ol & - & 0.1 & - \\
\hline 1714 & $\beta$-Davanon-2-ol & - & 0.4 & - \\
\hline 1714 & Pentadecanal & 0.2 & - & - \\
\hline 1728 & Oplopanone & $\operatorname{tr}$ & - & - \\
\hline 1750 & Unidentified & - & 2.2 & - \\
\hline 1919 & Methyl palmitate & - & $\operatorname{tr}$ & - \\
\hline 1962 & Palmitic acid & - & 1.7 & - \\
\hline 2029 & $(E, E)$-Geranyl linalool & 0.1 & - & 0.1 \\
\hline 2037 & $(Z)$-Falcarinol & - & $\operatorname{tr}$ & - \\
\hline 2105 & (E)-Phytol & 0.1 & 1.1 & - \\
\hline 2500 & Pentacosane & $\operatorname{tr}$ & - & - \\
\hline \multirow[t]{7}{*}{2700} & Heptacosane & $\operatorname{tr}$ & - & - \\
\hline & Total identified & 91.2 & 92.4 & 89.4 \\
\hline & Monoterpene hydrocarbons & 13.1 & 0.1 & 30.9 \\
\hline & Oxygenated monoterpenoids & 12.5 & 4.3 & 14.1 \\
\hline & Sesquiterpene hydrocarbons & 39.1 & 8.9 & 32.5 \\
\hline & Oxygenated sesquiterpenoids & 25.4 & 64.0 & 11.2 \\
\hline & Miscellaneous & 1.1 & 15.0 & 0.8 \\
\hline
\end{tabular}


${ }^{a} \mathrm{RI}=$ "Retention Index", determined with respect to a homologous series of $n$-alkanes on an HP$5 \mathrm{~ms}$ column.

${ }^{\mathrm{b}} \operatorname{tr}=$ "trace" $(<0.05 \%)$ 


\section{Figure Legend}

Fig. 1. Dendrogram obtained from the agglomerative hierarchical cluster analysis of 39 Lantana camara essential oil compositions from the literature [14-18,21-23,25-44] in addition to the compositions of the samples from Cuba, Nepal, and Yemen from this present study. 


\section{Lantana camara Dendrogram}

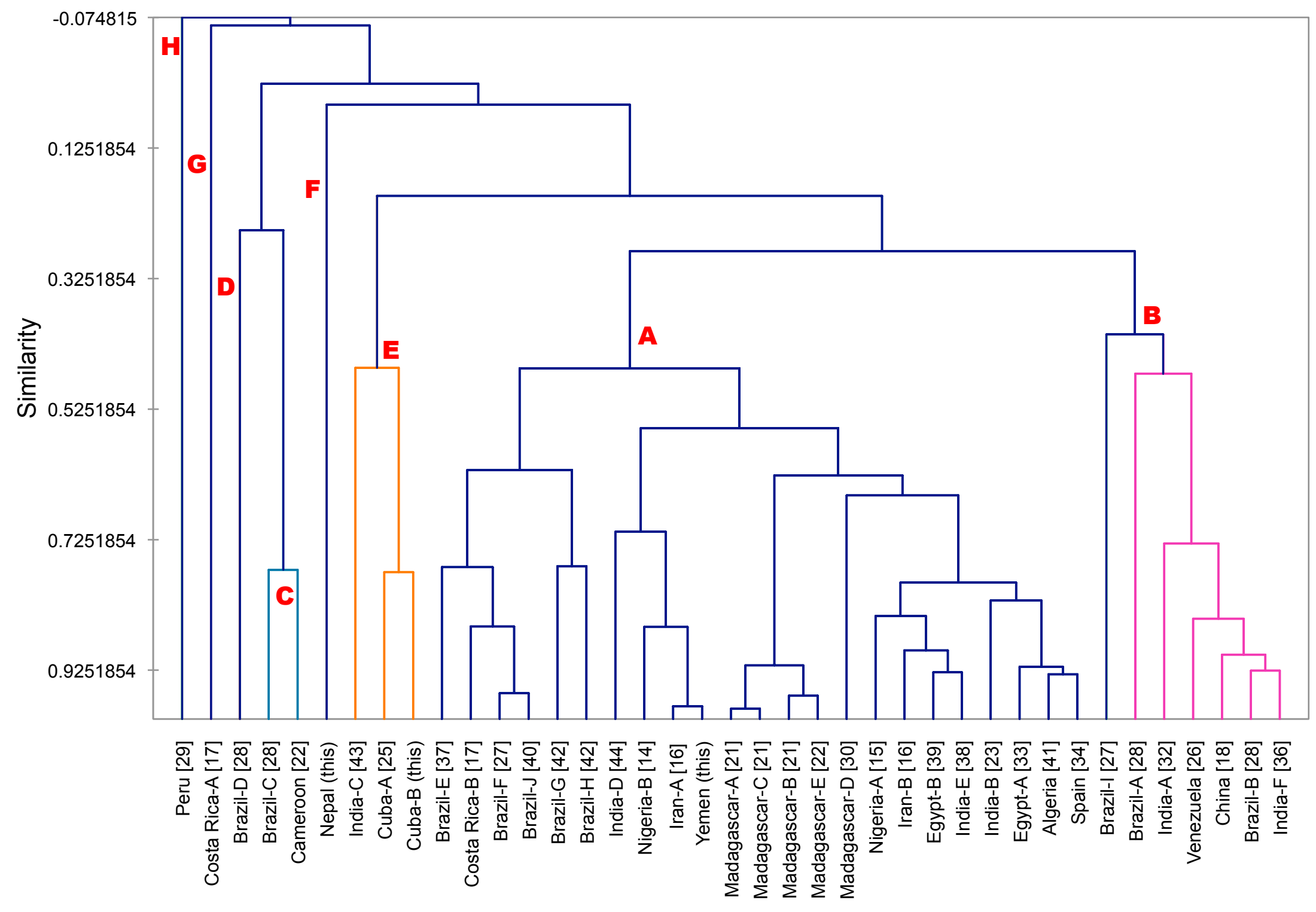

